Short Report

\title{
Application of Doppler Color Flow Imaging Method on the Detection of Vesicoureteral Reflux
}

\author{
Osamu Nishizawa, Hideaki Ishida, ${ }^{*}$ Kimio Sugaya, \\ Takeo Kohama, Tadashi Harada and Seigi Tsuchida \\ Department of Urology and *the First Department of \\ Internal Medicine, Akita University School of Medicine, \\ Akita 010
}

Nishizawa, O., Ishida, H., Sugaya, K., Kohama, T., Harada, T. and Tsuchida, S. Application of Doppler Color Flow Imaging Method of the Detection of Vesicoureteral Reflux. Tohoku J. Exp. Med., 1989, 159 (2), 163-164 The doppler color flow imaging method was employed to detect the vesicoureteral reflux. The ultrasound examination was performed by using the doppler color flow imaging system. Ultrasonic sector scan was carried out by the use of the 3.75 $\mathrm{MHz}$ phased array and/or conbex array transducer with a transabdominal approach. The flow in the bladder from the ureteral orifice to the bladder was detected with a red color indicating the direction towards the probe and the flow from the bladder towards the ureteral orifice during the vesicoureteral reflux was detected with a blue color indicating the direction away from the probe. The doppler color flow imaging method appears to be a useful diagnostic procedure for the detection of the vesicoureteral reflux.—- color doppler; vesicoureteral reflux; detection

A doppler color flow imaging method has recently been used for the detection of the both arterial and venous blood streams. Having been improved, this imaging system can now detect the minimum low flow speed of about $5 \mathrm{~mm} / \mathrm{sec}$ (Machi 1989). There is increaseing interest in the application of the doppler color flow imaging method on the urodynamics studying the mechanism of the urine transport from the kidney to the urethra with a relatively slow speed. In the previous report by Hashimoto et al. (1989), the flows from the ureteral orifice to the bladder and from the internal urethral orifice to the urethra have been detected by the doppler color flow imaging method. The present study was attempted to detect the vesicoureteral reflux flow using the doppler color flow imaging method.

A five year old girl was first hospitalized with fever elevation and urethral pain when she was four year old. An evaluation at that time showed the right grade 3 vesicoureteral reflux and neurogenic bladder due to spina bifida. Since then, intermittent catheterization was performed by her mother four times a day. However, recurrent urinary tract infection occurred, and the right hydronephrosis progressively advanced. At this time, patient was referred to our hospital for further evaluation of the vesicoureteral reflux and renal function.

The cystogram in the supine position revealed grade 4 vesicoureteral reflux on the right side at $200 \mathrm{ml}$ of the bladder capacity and the ureteral dilatation with caliecectasis and

Received July 17, 1989; revision accepted for publication September 27, 1989. 


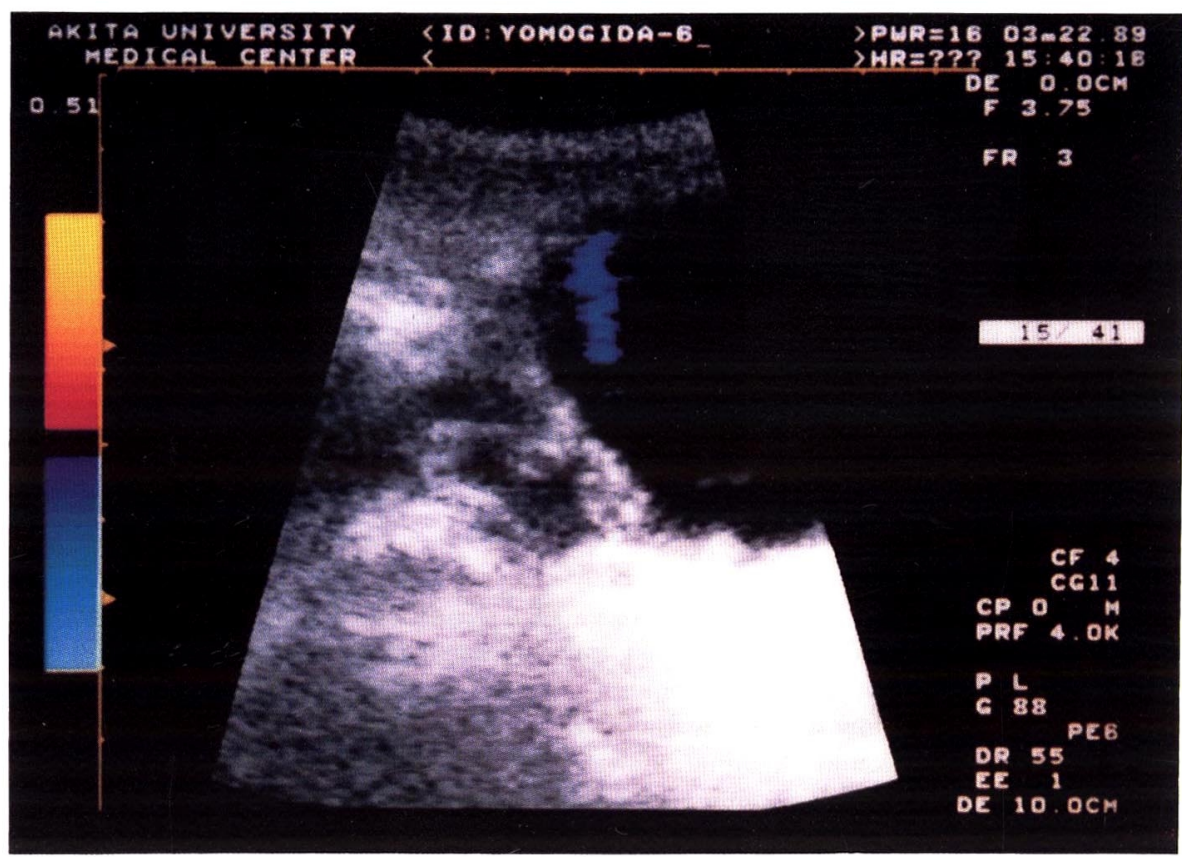

Fig. 1. The flow from the bladder towards the ureteral orifice is detected with a blue color.

blunting of calices. The doppler color flow imaging method was applied to detect a urine flow around the ureteral orifice. An ultrasound examination was performed by using the color doppler imaging system (SSA-270A ; Toshiba, Tokyo). Frequency of the transducer was $3.75 \mathrm{MHz}$. Ultrasonic sector scan of the bladder was carried out by the use of phased array and/or Convex array transducer with a transabdominal approach. The flow towards the probe was displayed with a red color. On the other hand the flow away from the probe was displayed with a blue color. At the examination, the patient was examined in the supine position with the bladder volume of about $200 \mathrm{ml}$ identical to those developing a vesicoureteral reflux at the time of the cystogram.

Fig. 1 shows the flow from the bladder towards the ureteral orifice during the vesicoureteral reflux with a blue color indicating the direction away from the probe. The substance in the urine to reflect ultrasound and the mechanism of detection of the urine flow by ultrasound is still unknown. The future development of the ultrasound equipment having higher sensitivity for the slower flow appear to make the doppler color flow imaging method a more effective diagnostic procedure for the detection of the vesicoureteral reflux.

\section{References}

1) Hashimoto, T., Saitoh, M., Matsuda, T., Kobayashi, H., Saitoh, T. \& Watanabe, H. (1989) Color doppler imaging in urodynamics (1st report) - Jet phenomenon of urine from the ureteral orifice and uroflow in the lower urinary tract-. Jan. J. Med. Ultrasonics, 16, Suppl. I, 575-576.

2) Machi, J. (1989) Color doppler imaging system. Jpn. J. Med. Ultrasonics, 16, 98-99. 